OPEN ACCESS

Edited by:

Alexander D. Borowsky,

University of California, Davis,

United States

Reviewed by:

Michele Bernasconi,

University Children's Hospital Bern,

Switzerland

Huarong Chen,

The Chinese University of Hong Kong,

China

Ruth Birbe,

Cooper Medical School of Rowan University, United States

*Correspondence:

Wei Yu

Wei-yu@zju.edu.cn

Bin Yuan

yuanbin@gwu.edu

Specialty section:

This article was submitted to

Molecular Medicine,

a section of the journal

Frontiers in Cell and Developmental

Biology

Received: 02 October 2020

Accepted: 15 January 2021

Published: 04 February 2021

Citation:

Wang Z, Yang Q, Tan Y, Tang Y,

Ye J, Yuan B and Yu W (2021)

Cancer-Associated Fibroblasts

Suppress Cancer Development:

The Other Side of the Coin.

Front. Cell Dev. Biol. 9:613534.

doi: 10.3389/fcell.2021.613534

\section{Cancer-Associated Fibroblasts Suppress Cancer Development: The Other Side of the Coin}

\author{
Zhanhuai Wang', Qi Yang'2, Yinuo Tan ${ }^{3}$, Yang Tang ${ }^{1}$, Jun Ye ${ }^{4}$, Bin Yuan ${ }^{5 *}$ and Wei Yu6* \\ ${ }^{1}$ Department of Colorectal Surgery and Oncology, Key Laboratory of Cancer Prevention and Intervention, Ministry \\ of Education, The Second Affiliated Hospital, Zhejiang University School of Medicine, Hangzhou, China, ${ }^{2}$ Department \\ of Pathology, The Second Affiliated Hospital, Zhejiang University School of Medicine, Hangzhou, China, ${ }^{3}$ Department \\ of Medical Oncology, Key Laboratory of Cancer Prevention and Intervention, Ministry of Education, The Second Affiliated \\ Hospital, Zhejiang University School of Medicine, Hangzhou, China, ${ }^{4}$ Department of Gastroenterology, The Second Affiliated \\ Hospital, Zhejiang University School of Medicine, Hangzhou, China, ${ }^{5}$ Department of Biochemistry and Molecular Medicine, \\ School of Medicine and Health Sciences, The George Washington University, Washington, DC, United States, ${ }^{6}$ Department \\ of Radiation Oncology, Key Laboratory of Cancer Prevention and Intervention, Ministry of Education, The Second Affiliated \\ Hospital, Zhejiang University School of Medicine, Hangzhou, China
}

Cancer-associated fibroblasts (CAFs) are the main stromal components of cancer, representing a group of heterogeneous cells. Many studies indicate that CAFs promote tumor development. Besides, evidence of the tumor suppression effects of CAFs keeps on merging. In the tumor microenvironment, multiple stimuli can activate fibroblasts. Notably, this does not necessarily mean the activated CAFs become strong tumor promoters immediately. The varying degree of CAFs activation makes quiescent CAFs, tumor-restraining CAFs, and tumor-promoting CAFs. Quiescent CAFs and tumorrestraining CAFs are more present in early-stage cancer, while comparatively, more tumor-promoting CAFs present in advanced-stage cancer. The underlying mechanism that balances tumor promotion or tumor inhibition effects of CAFs is mostly unknown. This review focus on the inhibitory effects of CAFs on cancer development. We describe the heterogeneous origin, markers, and metabolism in the CAFs population. Transgenetic mouse models that deplete CAFs or deplete CAFs activation signaling in the tumor stroma present direct evidence of CAFs protective effects against cancer. Moreover, we outline CAFs subpopulation and CAFs derived soluble factors that act as a tumor suppressor. Single-cell RNA-sequencing on CAFs population provides us new insight to classify CAFs subsets. Understanding the full picture of CAFs will help translate CAFs biology from bench to bedside and develop new strategies to improve precision cancer therapy.

Keywords: cancer-associated fibroblasts, neoplasms, tumor microenvironment, biomarker, Humans

Abbreviations: CAFs, cancer-associated fibroblasts; CAC, colitis associated-colon cancer; DNMT1, DNA (cytosine-5)methyltransferase 1; Evs, extracellular vesicles; HCC, hepatocellular carcinoma; HGF, hepatocyte growth factor; HR, hormone receptor; IHC, Immunohistochemistry; ISH, in situ hybridization; NFs, normal fibroblasts; OSCC, oral squamous cell carcinoma; PDAC, pancreatic ductal adenocarcinoma; TNBC, triple-negative breast cancer. 


\section{INTRODUCTION}

Fibroblasts are one of the significant stromal components in many organs, e.g., the gastrointestinal tract. They participate in adjacent tissue components' function through paracrine signaling and juxtacrine signaling (Biswas et al., 2015; Kalluri, 2016). One of their most crucial tasks is regulating ECM synthesis. When tissue injury happens, fibroblasts are getting ready to respond. They actively act to restrain the injury place and reconstruct the wound tissue framework. The classic description of cancer is "wounds that never heal." Could fibroblasts act as potential defenders against cancer progression? Numerous studies are being carried out to answer this question, but the results are unclear or sometimes contradicting. Generally, fibroblasts observed within and adjacent to the cancer are termed as cancer-associated fibroblasts (CAFs). In practice, the criteria to define CAFs are (1) mesenchymal cell isolated from a tumor; (2) negative markers for epithelial cells, endothelial cells and leukocyte markers; (3) elongated morphology; and (4) exclude the mutations of cancer cells (Sahai et al., 2020). CAFs are a group of heterogeneous cells and are the most prevalent stromal components of the tumor microenvironment. Previous studies have focused on tumor cells, yet now, it is increasingly recognized that the stromal components of the tumor microenvironment play critical roles in cancer progression (Hanahan and Weinberg, 2011). Importantly, CAFs play diverse parts in the interaction between different components in the tumor microenvironment. Thus, in cancer biology, understanding the functional evolution of CAFs during cancer development becomes a fundamental question to answer.

In the tumor microenvironment, potential stimuli that activate fibroblasts include bone morphogenetic protein (BMP), interleukin-1, interleukin-6, platelet-derived growth factor (PDGF), sonic hedgehog $(\mathrm{SHH})$, reactive oxygen species (ROS), transforming growth factor- $\beta$ (TGF- $\beta$ ), as well as TNF. These stimuli are released by tumor cells and multiple stromal components, including CAFs themselves (Theiss et al., 2005; Erez et al., 2010; Kalluri, 2016). The activated fibroblasts specifically express $\alpha$ smooth muscle actin ( $\alpha$-SMA), fibroblast activation protein (FAP), fibroblast-specific protein 1 (FSP1), PDGF receptor- $\beta$ (PDGFR $\beta$ ), and some other myofibroblasts markers. $\alpha$-SMA, a cytoskeleton protein, functions as a cell contraction regulator, is most commonly used as an activated fibroblast marker (Ohlund et al., 2014; Kalluri, 2016; Sahai et al., 2020). Cell morphology is a reliable way to distinguish activated CAFs within the quiescent fibroblasts. Activated CAFs undergo morphological changes from spindle-shaped into cruciform or stellate shaped. They become more proliferative, migrative, and metabolically active, with the enhanced acquisition of ECM production and synthetic phenotype. They have different epigenetic expression pattern compared to quiescent fibroblasts (Albrengues et al., 2015; Zhang et al., 2015; Kalluri, 2016). Notably, the activation of fibroblasts by stimuli in the tumor microenvironment does not necessarily mean these activated CAFs become strong cancer promoters immediately. Indeed, the varying degree of CAFs activation patterns produce quiescent CAFs, tumor-restraining CAFs, and tumor-promoting CAFs (Sugimoto et al., 2006; Kobayashi et al., 2019). Quiescent CAFs and tumor-restraining CAFs are frequently present in early-stage cancer, while comparatively, more tumor-promoting CAFs are predominantly present in advanced-stage disease. The chronic activation of CAFs requires continuous cross-talk between CAFs and cancer cells. This permanent cross-talk educates CAFs to accquire the pro-tumorigenic ability. Meanwhile, the stimuli that promote CAFs activation are essential factors that enhance tumor niche formation (Roberts et al., 2017). The co-evolution of CAFs and cancer cells continues throughout all the cancer progression stages (Figures 1, 2).

The tumor microenvironment plays a key part in fostering tumor progression through the collaboration of multiple components. Besides, evidence of the tumor inhibition effects of individual cancer stromal component keeps on merging. The underlying mechanism that balances tumor promotion or tumor inhibition effects of the tumor microenvironment is mostly unknown. Most studies that explore CAFs functions show that CAFs support tumor growth. Naturally, the reverse side evidence of this is much less. If the tumor stroma inhibiting effect continuously exists, the tumor can not necessarily develop. Failure to educate quiescent fibroblasts to acquire protumorigenic ability means tumor cells might not survive (Mueller and Fusenig, 2004; Proia and Kuperwasser, 2005). In the very beginning of tumorigenesis, tumor cells conduct a dedicated education system to reprogram CAFs and other tumor stroma cells. In the later stage, tumor cells deliver messages to distant healthy organs stroma to build a pre-metastatic stromal niche (Hanahan and Weinberg, 2011). Recently, tumor associatedextracellular vesicles (EVs) have been identified as an important cellular interchange mechanism between tumor cells and CAFs (Becker et al., 2016; Maacha et al., 2019). EVs isolated from tumor cells and CAFs are implicated in multi-steps of CAFs evolution, such as normal fibroblasts (NFs) differentiation into CAFs, CAFlike state maintenance, and CAFs' function support (Luga et al., 2012; Webber et al., 2015; Richards et al., 2017; Giusti et al., 2018).

Understanding the time point when CAFs shift from tumor defender into tumor supporter is critically essential. Thus, preventing CAFs functional change from a tumor-supportive phenotype will be potentially promising in anti-tumor therapy. This review focus on the inhibitory effects of CAFs on cancer development. We describe the heterogeneous origin, markers, and metabolism in the CAFs population. Transgenetic mouse models that deplete CAFs or deplete CAFs activation signaling in the tumor stroma present direct evidence of CAFs' protective effects against cancer. Moreover, we outline CAFs subpopulation and CAFs derived soluble factors that act as a tumor suppressor. Single-cell RNA-sequencing on CAFs population provides us new insight to classify CAFs subsets. The main findings concerning the tumor inhibitory effects of CAFs are list in Table $\mathbf{1}$.

\section{THE HETEROGENEITY OF CAFS POPULATION}

\section{Heterogenous CAFs Origin}

Despite the highly organized NFs, the CAFs population is highly heterogeneous. The origin of CAFs is still contested. The direct correlation between CAFs subtype function and CAFs subtype 


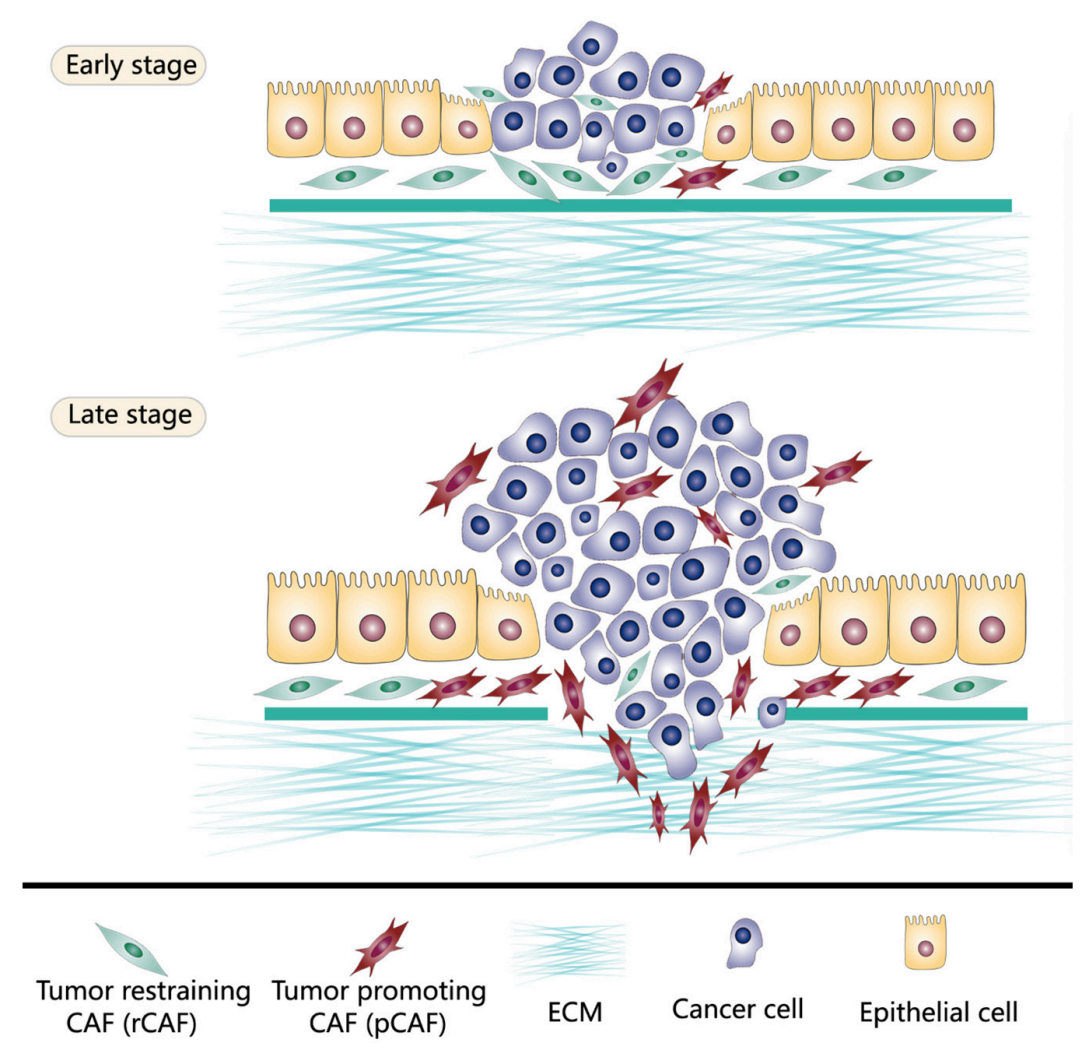

FIGURE 1 | Tumor-restraining CAFs (rCAFs) and tumor-promoting CAFs (pCAFs) both exist in the tumor microenvironment. rCAFs are frequently present in early-stage cancer which protect normal tissue against cancer invasiveness. However, in advanced-stage disease, tumor cells reprogram CAFs through continuous messages exchange to build cancer supporting stromal niche. Thus, pCAFs are predominantly present in late-stage cancer.

cellular origin is not clearly illustrated. However, it is convincing that CAFs originated from different cell lineages might have distinct functional phenotypes (Ishii et al., 2015; Sahai et al., 2020). In general, CAFs have four main sources of cellular origin. The primary source is normal local fibroblasts, which are activated by stimuli from the tumor microenvironment. Mesenchymal stem cells (MSCs) and other mesenchymal precursor cells are other sources. They are recruited to the TME to become CAFs-state cells by cytokines and chemokines, including TGF- $\beta$ and CXC-chemokine ligand 12 (CXCL12; Koliaraki et al., 2017). Endothelial cells and epithelial cells do not belong to the fibroblast lineage, but they could transdifferentiate into CAFs-state cells. Finally, a self-renewable CAFs-stem cell population might exist in the hierarchical organization, and these cells share similar characteristics as MSCs. They differentiate into progeny CAFs including cancer-promoting CAFs and cancerrestraining CAFs (Worthley et al., 2015).

\section{Heterogenous CAFs Marker}

Studies to identify CAF subsets are still in their infancy. In the experiment, CAFs are isolated through flow cytometrybased cell sorting by using a series of combined markers. The markers that CAFs lack expression of are CD31 (an endothelial marker), CD45 (a hematopoietic cell marker), desmin (a smooth muscle cell marker), and EPCAM (epithelial cell adhesion molecule, an epithelial cell marker). These markers are combined with a representative CAFs marker (e.g., FAP) for CAFs sorting (Feig et al., 2013; Calon et al., 2015; Costa et al., 2018). The most commonly used CAFs markers include but are not limited to $\alpha$-SMA, Collegen1A1, FAP, FSP1, PDGFR $\alpha$ and PDGFR $\beta$, Podoplanin, and vimentin. However, all of these markers are not CAFs specific and can be expressed in other cell types in cancer or normal tissues (Kalluri, 2016). $\alpha$-SMA is unable to identify all CAFs in the TME, and it is expressed in smooth muscle in normal gastrointestinal and vascular (Koliaraki et al., 2017; Ohlund et al., 2017). FAP combined with CD45 is used to label a subgroup of cancer-associated macrophages (Arnold et al., 2014). FSP1 also corresponds to epithelial cells experiencing epithelial-to-mesenchymal transition (EMT; Rhim et al., 2012; Fischer et al., 2015). In a liver fibrosis model, FSP1 distinguishes inflammatory macrophage subpopulation (Osterreicher et al., 2011). The different origins of CAFs add to the complexity of CAFs definitions. A sole CAFs marker is impossible to find theoretically. Depending on different markers used, different results regarding CAFs pro-tumorigenic function ( $\mathrm{pCAFs)}$ or CAFs tumor-restain (rCAFs) function can be obtained (Kobayashi et al., 2019). One of the most urgent works required in CAFs biology is to explore the different markers of subtype CAFs based on biology and function. 


\section{Tumor Microenvironment}

Activation Stimuli

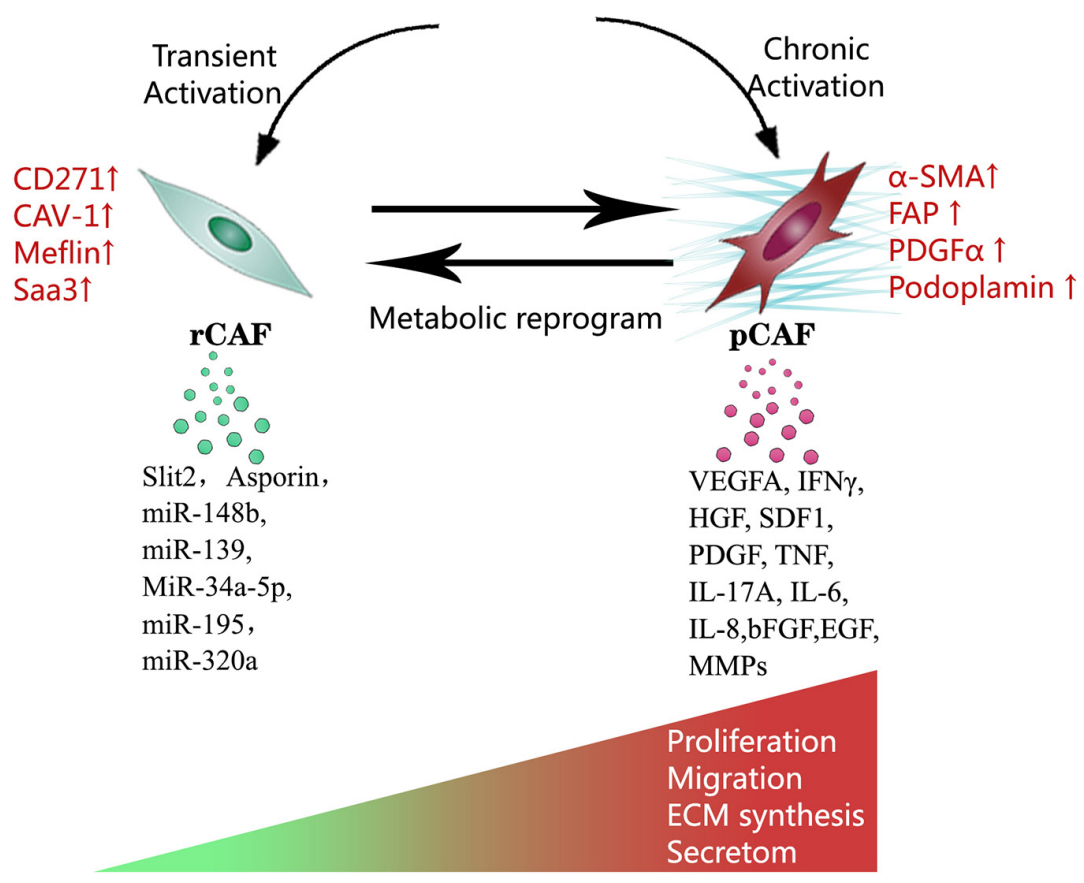

FIGURE 2 | Multiple stimuli in the tumor microenvironment activate CAFs. Notably, this activation process does not necessarily mean the activated CAFs become strong cancer promoters immediately. Depending on the degree of activation, CAFs can be functionally divided into tumor-restraining CAFs, and tumor-promoting CAFs. Transient activation makes tumor-restraining CAFs and chronic activation makes tumor-promoting CAFs. Tumor-restraining CAFs are morphologically similar to quiescent fibroblasts. Tumor-promoting CAFs undergo morphological changes from spindle-shaped into cruciform or stellate shaped. Two types of CAFs show the main difference in cellular markers, metabolic status, proliferation, migration, ECM synthesis, and secretory phenotype.

\section{Heterogenous CAFs Metabolism}

Traditionally, the glycolytic pathway has been considered the main metabolic pathway in cancer. Recent studies revealed that cancers are highly organized tissues and are categorized with heterogeneous metabolism in different components (Wallace, 2012). Even in the CAFs population, the heterogeneous metabolic status also exists. Compared to quiescent fibroblasts, parts of activated CAFs undergo metabolic changes and become catabolic CAFs. Biomarkers of this catabolic CAFs phenotype including down-regulated caveolin-1 (Cav-1; Martinez-Outschoorn et al., 2011) and up-regulated monocarboxylate transporter 4 (MCT4; Whitaker-Menezes et al., 2011). Cav-1 is abundantly expressed in NFs. Loss of Cav-1 expression is an autophagy marker, indicating decreased mitochondrial metabolisms, such as oxidative phosphorylation (OXPHOS), increasing glycolysis and oxidative stress (Sotgia et al., 2011). MCT4, with its expression controlled by HIF-1, is the main cellular lactate exporter (Ullah et al., 2006). Recent studies demonstrate the collaboration of catabolic fibroblasts and anabolic cancer cells, which is the so-called metabolic coupling. This metabolic coupling exists in many different human malignancies, such as breast cancer, head and neck cancer, and prostate cancer (Giatromanolaki et al., 2012; Witkiewicz et al., 2012; Curry et al., 2013). The driving pathway of catabolic CAFs are HIF1- $\alpha$, NFкB signaling, and TGF- $\beta$ signaling, promoting autophagy, glycolysis, oxidative stress, and senescence (Martinez-Outschoorn et al., 2014). These catabolic CAFs generate local mitochondrial fuels, such as fatty acids, glutamine, ketone bodies, lactate, to support the cancer microenvironment (Martinez-Outschoorn et al., 2014). Noticed that not all the CAFs become catabolic CAFs, the catabolic CAFs and the anabolic CAFs both exist. The catabolic changes of CAFs could be reversed upon anti-oxidants treatments (Yang et al., 2016; Monti et al., 2017; Zhang et al., 2018). Does the transformation from tumor-restraining CAFs into tumor-promoting CAFs accompany with catabolic phenotype changes? The answer is still not clear. Explore the heterogeneity in CAFs metabolism will help us to classify CAFs in a critical functional aspect.

\section{THE PROTECTIVE ACTION OF CAFS AGAINST CANCER: EVIDENCE FROM TRANSGENIC MICE}

\section{Depletion of CAFs Enhances Tumor Development}

The transgenic mouse model allows us to deplete CAFs through germline mutation or organ-specifically in mature tissue. Such a model helps to investigate roles that CAFs 
TABLE 1 | Studies focus on the tumor inhibitory effects of CAFs.

\begin{tabular}{|c|c|c|}
\hline Author & Cancer type & Main findings \\
\hline Dai et al., 2019 & $\begin{array}{l}\text { Mice pancreatic } \\
\text { cancer }\end{array}$ & $\begin{array}{l}1 \text { In RAMP3-/- mice, spleen injection of PAN02 murine pancreatic cancer cells showed reduced liver metastasis. } \\
2 \text { RAMP3-/- mice metastatic tumor showed decreased podoplanin positive or } \alpha \text {-SMA positive CAFs. } \\
3 \text { Primary RAMP3-/- CAFs inhibited proliferation, migration, and metastasis in co-cultures with PAN02 murine pancreatic } \\
\text { cancer cells. }\end{array}$ \\
\hline Mizutani et al., 2019 & $\begin{array}{l}\text { Mice Pancreatic } \\
\text { ductal } \\
\text { adenocarcinoma } \\
\text { (PDAC) }\end{array}$ & $\begin{array}{l}1 \text { Meflin is expressed in CAFs originate from pancreatic stellate cells } \\
2 \text { Meflin positive CAFs showed decreased a-SMA expression and stromal collagen regulating } \\
3 \text { Meflin negative CAFs showed more aggressive pro-tumorigenic functions } \\
4 \text { Meflin knockout mice showed a poorly differentiated tumor with more a-SMA + CAFs compared to Meflin wild type mice }\end{array}$ \\
\hline Djurec et al., 2018 & Mice PDAC & $\begin{array}{l}1 \text { PDGFR } \alpha+\text { Saa3 + CAFs stimulated mice PDAC growth, but PDGFR } \alpha+\text { Saa3- CAFs inhibited tumor growth. } 2 \text { The } \\
\text { PDGFR } \alpha+\text { Saa3- CAFs inhibited tumor growth by overexpression Mpp6 }\end{array}$ \\
\hline Gerling et al., 2016 & $\begin{array}{l}\text { Mice colitis } \\
\text { associated-colon } \\
\text { cancer (CAC) }\end{array}$ & $\begin{array}{l}1 \text { Downstream Hh signaling is restricted to the tumor stroma particularly in CAFs } \\
2 \text { Hh signaling deletion in CAFs promoted tumorigenesis, whereas Hh activation inhibited tumor progression } \\
3 \text { Hh signaling in CAFs suppressed tumor by regulating BMP activity and inhibting colonic stem cell signature }\end{array}$ \\
\hline Maris et al., 2015 & Breast cancer & $\begin{array}{l}1 \text { Asporin suppressed TGF- } \beta 1 \text {-mediated SMAD2 phosphorylation, EMT, and cancer stem cell signature. } \\
2 \text { Asporin overexpression CAFs reduce tumor growth in the mice xenograft TNBC model }\end{array}$ \\
\hline Koliaraki et al., 2015 & Mice CAC & $\begin{array}{l}\text { Genetic deletion of IKK } \beta \text { in COLVI + CAFs caused decreased tumor growth and inflammation in the CAC mice model. The } \\
\text { effect is mainly due to the down-regulated IL-6 release in IKK } \beta \text {-deficient CAFs compared to control }\end{array}$ \\
\hline Pallangyo et al., 2015 & Mice CAC & $\begin{array}{l}1 \text { Genetic deletion of IKK } \beta \text { in COL1A2 + CAFs in mice colon cancer accelerated tumor growth } \\
2 \text { IKK } \beta \text {-deficient COL1A2 + CAFs showed enhanced secretion of HGF, which promoted tumor growth through HGF-Met } \\
\text { signaling }\end{array}$ \\
\hline Ozdemir et al., 2014 & Mice PDAC & $\begin{array}{l}1 \text { Effects of genetic a-SMA + myofibroblast depletion were test in both early and late stage PDAC } \\
2 \text { a-SMA + myofibroblast depletion tumor showed more invasive, undifferentiated, and necrotic characteristics with a poor } \\
\text { survival compared to control. }\end{array}$ \\
\hline Rhim et al., 2014 & Mice PDAC & $\begin{array}{l}1 \mathrm{SHH} \text {-deficient PDAC showed decreased a-SMA-positive myofibroblasts compared to the control tumor. } \\
2 \mathrm{SHH} \text {-deficient PDAC exhibited undifferentiated histology, increased proliferation, vascularity, and reduced survival time. }\end{array}$ \\
\hline Zhang et al., 2013 & $\begin{array}{l}\text { Mice skin } \\
\text { fibrosarcoma }\end{array}$ & $\begin{array}{l}1 \text { FSP1 was predominantly expressed in procollagen I + fibroblasts in mice skin } \\
2 \text { Genetic deletion of FSP1 + CAFs enhanced mice skin fibrosarcoma formation }\end{array}$ \\
\hline Chang et al., 2012 & Breast cancer & 1 CAFs derived Slit2 conducted its tumor inhibition effects by bind to Robo1 receptor expressed in cancer cells. \\
\hline
\end{tabular}

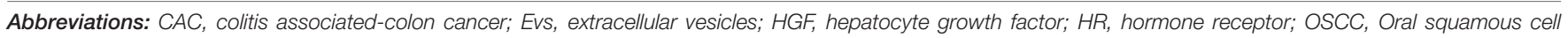
carcinoma; PDAC, Pancreatic ductal adenocarcinoma; and TNBC, triple-negative breast cancer.

play in different stages of carcinogenesis. What happens if CAFs are diminished in certain types of tumors? Results of recent studies strikingly indicated that CAFs depletion causes rapid tumor progression rather than tumor suppression. a-SMA is one of the most commonly used CAFs markers, distinguishing activated fibroblasts from the quiescent fibroblasts in tumor microenvironments (Kalluri, 2016). Ozdemir et al., applied a genetic approach to deplete a-SMA + myofibroblasts in mice selectively. They aimed to examine the function of a-SMA + myofibroblasts in early-stage, as well as latestage pancreatic ductal adenocarcinoma (PDAC). They crossed a-SMA- thymidine kinase (TK) transgenic mice that allowed target depletion of a-SMA + myofibroblasts and the mice that developed spontaneous PDAC. By crossed two type of mice, a-SMA + myofibroblasts could be selectively depleted in PDAC tissue. The effects of a-SMA + myofibroblasts depletion were analyzed in both early-stage and late-stage PDAC. Compared to control, a-SMA + myofibroblast depletion tumor was significantly more invasive, undifferentiated, and necrotic. Mice bearing a-SMA + myofibroblast depletion tumor ended up with multiple adverse outcomes. The author also suggested that a-SMA + myofibroblast produced collagen I and the associated fibrosis protected the host against tumorigenesis at both early and late stages of PDAC (Ozdemir et al., 2014). Zhang et al. tested the hypothesis of whether depletion FSP1 fibroblasts impair skin fibrosarcoma formation. The author applied FSP-TK transgenic mice, which allowed FSP1 + cells to be selectively depleted upon administration of ganciclovir (GCV; Salomon et al., 1995; Iwano et al., 2001). Zhang et al. found that FSP1 was predominantly expressed in procollagen I + fibroblasts in mice skin. Thus, FSP1 + depletion in stromal cells mainly caused CAFs depletion. Mice model of skin fibrosarcoma was induced via carcinogen methylcholanthrene (MCA) subcutaneous injection. A Ablation of FSP1 + cells enhanced tumor formation and altered skin fibrosarcoma morphology. Interestingly, these skin tumors showed epithelial phenotype instead of fibroblastoid. The author also showed that degrading collagen produced by fibroblasts promoted tumor formation in the long-term "tumorfree" mice. These findings indicated that FSP1 + CAFs and collagens exert a critical protective role against chemical induced fibrosarcoma in the skin (Zhang et al., 2013).

\section{Depletion of Activation Signaling in CAFs Enhances Tumor Progression}

Cancer-associated fibroblasts activation signaling relies on various stimuli from the TME, and $\mathrm{SHH}$ is one of these. $\mathrm{SHH}$, a soluble ligand of hedgehog signaling, is frequently overexpressed by neoplastic cells, which stimulates CAFs to form a fibroblast-rich desmoplastic stroma (Valenti et al., 2017). Earlier studies indicated that over activation of hedgehogsignaling accelerated tumorigenesis in the pancreatic epithelium 
(Mao et al., 2006; Pasca Di Magliano et al., 2006; Morton et al., 2007). Since the deletion of $\mathrm{SHH}$ expression might impair CAFs activation signaling, what is the impact on malignant progression if $\mathrm{SHH}$ expression is removed from cancer cells? Rhim et al used the transgenic mice to delete $\mathrm{SHH}$ in the PDAC model. The SHH-deficient tumor showed decreased stromal content, especially a-SMA-positive myofibroblasts, compared to the control tumor. These tumors exhibited more aggressive features with undifferentiated histology, increased proliferation, and vascularity. SHH deletion also caused more frequent acinar-to-ductal metaplasia (ADM) and pancreatic intraepithelial neoplasia (PanIN) at a younger age. Thus, $\mathrm{SHH}$ deletion lead to rapid death in mice. $\mathrm{SHH}$ deletion in pancreatic cancer showed increased Zeb1 and Slug expression consistent with EMTs and increased metastasis (Rhim et al., 2014). Similar results were reported in a mouse model of colitis-associated colonic tumorigenesis. In that study, Gerling et al applied hedgehog $(\mathrm{Hh})$ signaling reporter mice. They demonstrated that Hh signaling deletion promoted tumorigenesis, whereas stromaspecific Hh activation significantly attenuated tumor progression. Activated $\mathrm{Hh}$ signaling in CAFs suppressed tumor growth through regulating BMP activity and inhibiting colonic stem cell gene expression (Gerling et al., 2016). The IKK $\beta$-dependent NF- $\mathrm{KB}$ signaling activation is considered as a key to connect inflammation and carcinogenesis (Greten et al., 2004). CAFs from the cervical, mammary, pancreatic, and skin tumors exhibit a proinflammatory signature regulated by $N F-\kappa B$ signaling (Erez et al., 2010). Two separate studies probed the effects of IKK $\beta$ on CAFs during tumor development. The first study used Tg (CollagenVI-Cre) mice, which allowed tracing of the distribution of collagen type VI positive (COLVI + ) fibroblasts in the mice intestine. Tg (CollagenVI-Cre) mice were crossed with $\operatorname{Ikk} \beta^{\mathrm{F} / \mathrm{F}}$ mice to generate IKK $\beta$ deficient COLVI + fibroblasts. These transgenic mice were further applied with AOM/DSS to form colitis-associated cancer (CAC). IKK $\beta$ deficiency in COLVI + CAFs caused decreased tumor growth and inflammation in the CAC mice model. The effect is mainly due to the down-regulated IL-6 release in IKK $\beta$-deficient CAFs compared to control (Koliaraki et al., 2015). The second study, however, showed the tumor-restraining function of IKK $\beta / N F$ $\kappa \mathrm{B}$ in CAFs. Koliaraki et al used a different Cre, collagen type a2 Cre (Colla2-creER), to trace fibroblasts. And Ikk $\beta F / F$ mice were crossed with Colla2-creER mice. COL1A2 + fibroblasts represent a larger population than COLVI + fibroblasts. The genetic deletion of IKK $\beta$ in COL1A2 + CAFs in the similar CAC model unexpectedly accelerated tumor growth. The Ikk $\beta$ deficient CAFs showed impaired Smad7 and Smurf1, which were both TGF- $\beta$ pathway negative regulators. Thus, the upregulation of TGF- $\beta$ signaling was observed in $\operatorname{Ikk} \beta$ deficient CAFs. Moreover, these CAFs showed enhanced secretion of hepatocyte growth factor (HGF), which promoted tumor growth through HGF-Met signaling (Pallangyo et al., 2015). Since COL1A2 targets nearly $80 \%$ of PDGFR $\alpha+$ fibroblasts, COL1A2 + fibroblasts comprised a much larger fibroblasts population than COLVI + fibroblasts. The possible explanation of conflict results is that different fibroblast subpopulations may exhibit distinct IKK $\beta / \mathrm{NF}-\kappa \mathrm{B}$ signaling functions. Meanwhile,
CAFs display different activation status could display different $\mathrm{NF}-\kappa \mathrm{B}$ signaling status.

\section{CAFS SUBPOPULATION INHIBIT TUMOR PROGRESSION}

Despite numerous results indicating CAFs' pro-tumorigenic effects, here, we provide shreds of evidence that CAFs subsets restrain tumor progression. Djurec et al., showed that PDGFR $\alpha+$ CAFs derived from a PDAC mouse model promoted tumor cell growth, and that normal pancreatic fibroblasts inhibited tumor growth. When PDGFR $\alpha+$ CAFs were subdivided by the expression of Saa3, a protein belonging to the acute-phase serum amyloid A (SAA) apolipoprotein family, PDGFR $\alpha+$ Saa3- CAFs and PDGFR $\alpha+$ Saa3 + CAF showed different properties. PDGFR $\alpha+\mathrm{Saa} 3+\mathrm{CAFs}$ stimulated mice PDAC growth in both the orthotopic model and organoid cultures; however, PDGFR $\alpha+$ Saa3- CAFs inhibited tumor growth. The PDGFR $\alpha+$ Saa3- CAFs exerted their tumor inhibition effects by overexpressing membrane palmitoylated protein 6 (Mpp6). This study sheds light on the future direction of target therapy through the management of Mpp6 expression (Djurec et al., 2018). Dai et al found that RAMP3 deficient CAFs inhibited tumor growth and metastasis. RAMPs, receptoractivity-modifying proteins, are modulators of G-proteincoupled receptors, which function as tumor angiogenesis regulator and prognostic marker (Mclatchie et al., 1998; Fang et al., 2018; Mackie et al., 2019). In RAMP3-/- mice, spleen injection of PAN02 murine pancreatic cancer cells showed significantly reduced liver metastasis. Compared to wild-type mice, liver metastatic lesion in RAMP3-/- mice showed decreased podoplanin positive or $\alpha$-SMA positive CAFs. Moreover, primary cultured RAMP3-/- CAFs inhibited proliferation, migration, and metastasis in co-cultures with PAN02 murine pancreatic cancer cells in vitro and in vivo (Dai et al., 2019). Meflin is a mesenchymal stromal/stem cell marker and indicates their undifferentiated state. In pancreatic cancer, Meflin is expressed in CAFs originate from pancreatic stellate cells. Meflin positive CAFs showed decreased a-SMA expression and stromal collagen regulation, while Meflin negative CAFs showed more aggressive pro-tumorigenic functions. In the mouse PDAC model, Meflin knockout mice showed a poorly differentiated tumor with more a-SMA + CAFs compared to Meflin wild type mice. Results suggested Meflin served as a tumor-restraining CAFs marker in PDAC (Mizutani et al., 2019).

\section{CAFS DERIVED SOLUBLE FACTORS SUPPRESS TUMOR PROGRESSION}

Cancer-associated fibroblasts support cancer progression by secreting various soluble factors, whereas CAFs can also derive tumor restraining factors. In breast cancer, CAFs derived Slit2 acted as a tumor inhibitor. Slit2 exerted tumor suppression effects by binding to Robol receptor expressed in cancer cells. Stable Robo1-depletion in breast cancer cells abolished the CAFs tumor 
suppression effect in the orthotopic mouse model. Meanwhile, ectopic Robol overexpression in breast cancer cells enhanced CAFs related tumor suppression effect. The active Slit2/Robo1 signaling prevented $\beta$-catenin translocation into nuclei, which resulted in c-myc and cyclin D1 downregulation through the PI3K/Akt pathway (Chang et al., 2012). Asporin, a stromal secreted extracellular matrix protein, inhibited canonical TGF$\beta /$ Smad signaling by binding to TGF- $\beta 1$ (Kizawa et al., 2005). The expression of asporin in CAFs increased when exposed to gastric cancer cells. CAFs-derived asporin activated CD44Rac1 pathway in cancer cells and coordinated the co-invasion of these two types of cells (Satoyoshi et al., 2015). Interestingly, asporin functional studies in breast cancer is another story. Triple-negative breast cancer (TNBC) cells suppressed CAFs' asporin expression by secreting IL-1 $\beta$, while hormone receptor (HR) positive breast cancer induced CAFs asporin expression. In vitro studies on breast cancer cells indicated asporin suppressed TGF- $\beta 1$-mediated SMAD2 phosphorylation, EMT, and cancer stem cell signature. In the murine model of TNBC, asporin overexpression CAFs could significantly reduce tumor growth. The results of the two studies seem to conflict with each other. TGF- $\beta$ signaling has both tumor suppression and tumor promotion effects depending on the cancer type and cancer stage (Seoane and Gomis, 2017). Asporin is a TGF$\beta 1$ natural inhibitor, which might also have various services in different cancer types.

MicroRNAs are known as small molecular RNA, which bind to their target mRNAs and negatively modulate gene expression at the post-transcriptional level. MicroRNAs transferred by exosomes are a common way of communication connecting CAFs and tumor cells ( $\mathrm{Li}$ et al., 2018). Recently studies investigated the microRNA changes and their function in CAFs derived exosomes. Our previous review illustrates CAFs-derived microRNAs promote cancer development through a variety of approaches (Wang et al., 2017). During tumor progression, CAFs secreted tumor-promoting microRNAs show increased expression and CAFs secreted tumor-suppressing microRNAs are inhibited (Wang et al., 2017). Several studies indicated that tumor-suppressing microRNAs could strongly inhibit tumor development when they were re-expressed in CAFs. These CAFs derived tumor suppressor microRNAs include miR-195 in cholangiocarcinoma (Li L. et al., 2017), MiR-34a-5p in oral squamous cell carcinoma (OSCC; Li et al., 2018), miR-148b in endometrial cancer (Li et al., 2019), miR-139 in gastric cancer (Xu et al., 2019), and miR-320 in hepatocellular carcinoma (HCC; Zhang et al., 2017; Table 2).

\section{CAFS RELATED PROTEIN PROVIDE FAVORABLE CLINICAL OUTCOMES}

Many studies have addressed the clinical prognostic value of CAFs markers and CAFs derived factors. These findings include but are not limited to FAP expression in CAFs being related to reduced survival time in non-small cell lung cancer (NSCLC; Liao et al., 2013), podoplanin expression in CAFs related to reduced recurrence-free survival in NSCLC
TABLE 2 | CAFs derived tumor suppression microRNAs.

\begin{tabular}{|c|c|c|}
\hline Author & Cancer type & Main findings \\
\hline $\begin{array}{l}\text { Li et al., } \\
2019\end{array}$ & Endometrial cancer & $\begin{array}{l}1 \mathrm{MiR}-148 \mathrm{~b} \text { decreased in CAFs and } \\
\text { CAFs-derived exosomes compared to NFs } \\
2 \text { CAFs derived miR-148b transferred to } \\
\text { endometrial cancer cells by exosomes } \\
3 \text { MiR-148b overexpression in CAFs } \\
\text { suppressed endometrial cancer metastasis } \\
\text { in vitro and in vivo by directly binding to DNMT1 }\end{array}$ \\
\hline $\begin{array}{l}\text { Xu et al., } \\
2019\end{array}$ & Gastric cancer & $\begin{array}{l}1 \text { MiR-139 level was down-regulated in tumors } \\
\text { compared with adjcent normal tissues } \\
2 \text { Exosomal miR-139 in CAFs was reduced } \\
\text { compared to NFs } \\
3 \text { Exosomes shuttled miR-139 from fibroblasts } \\
\text { to cancer cells } \\
4 \text { MiR-139 overexpression in CAFs suppressed } \\
\text { cancer cells growth and metastasis by inhibiting } \\
\text { the expression of MMP11 }\end{array}$ \\
\hline $\begin{array}{l}\text { Li et al., } \\
2018\end{array}$ & OSCC & $\begin{array}{l}1 \mathrm{MiR}-34 a-5 p \text { in CAF-derived exosomes was } \\
\text { reduced compared to NFs } \\
2 \text { Fibroblasts derived exosomal miR-34a-5p } \\
\text { could transfer to OSCC cells } \\
3 \text { miR-34a-5p overexpression in CAFs inhibited } \\
\text { OSCC cells proliferation and metastasis by } \\
\text { binding to AXL in cancer cells }\end{array}$ \\
\hline $\begin{array}{l}\text { Li L. et al., } \\
2017\end{array}$ & Cholangiocarcinoma & $\begin{array}{l}1 \text { Cholangiocarcinoma cells and the adjoining } \\
\text { CAFs showed down-regulated miR-195 } \\
2 \text { EVs shuttled miR-195 from fibroblasts to } \\
\text { cancer cells } \\
3 \text { miR-195 overexpression in fibroblasts } \\
\text { suppressed growth and invasion of } \\
\text { cholangiocarcinoma cells } \\
4 \text { miR-195 loaded EVs inhibit tumor and } \\
\text { improve survival of in a rat model of } \\
\text { cholangiocarcinoma. }\end{array}$ \\
\hline $\begin{array}{l}\text { Zhang } \\
\text { et al., } 2017\end{array}$ & $\mathrm{HCC}$ & $\begin{array}{l}1 \text { miR-320a level was reduced in CAFs-derived } \\
\text { exosomes compared to NFs } \\
2 \text { Fibroblasts derived exosomal miR-320a could } \\
\text { transfer to HCC cells } \\
3 \text { Exosomal miR-320a binded to PBX3 in HCC } \\
\text { cells and inhibited their proliferation and } \\
\text { metastasis. } \\
4 \text { MiR-320a-PBX3 axis suppressed tumor } \\
\text { progression by inhibiting MAPK pathway } \\
\text { acitivation }\end{array}$ \\
\hline
\end{tabular}

Abbreviations: DNMT1, DNA (cytosine-5)-methyltransferase 1; Evs, extracellular vesicles; HCC, hepatocellular carcinoma; HGF, hepatocyte growth factor; HR, hormone receptor; OSCC, Oral squamous cell carcinoma; and TNBC, triplenegative breast cancer.

(Ito et al., 2012; Ono et al., 2013), PDGFR $\beta$ expression in CAFs related to reduced cancer-specific survival in breast cancer patients (Paulsson et al., 2009). The correlation between particular CAFs related molecular and cancer prognosis, to some extent, reflect the education process of CAFs during tumor progression. Importantly, tumor-restraining CAFs also have particular markers and derived factors, and the increased expression of such molecules in patient samples indicates a better prognosis (Table 3). The membrane protein CAV-1 is associated with cell metabolism, modulating autophagy, cholesterol distribution, fatty acid metabolism, glycolysis, glutaminolysis, and mitochondrial bioenergetics. In CAFs, CAV-1 acts as a tumor suppression protein. CAFs that lose 
TABLE 3 | CAFs related protein provide favorable clinical outcomes: main findings.

\begin{tabular}{|c|c|c|c|c|}
\hline References & Gene & $\begin{array}{l}\text { Human } \\
\text { cancer type }\end{array}$ & $\begin{array}{l}\text { Methods and } \\
\text { patient number }\end{array}$ & Histopathological findings \\
\hline Rhim et al., 2014 & Meflin & $\begin{array}{l}\text { Pancreatic } \\
\text { cancer }\end{array}$ & $\mathrm{ISH}(n=71)$ & $\begin{array}{l}1 \text { Aproximately } 10 \% \text { of a-SMA + CAFs show positive Meflin mRNA expression in ISH assay. } \\
2 \text { Meflin-high ( } \geq 20 \% \text { Meflin }+ \text { stromal cells) group showe prognosis and a more differentiated histology } \\
\text { than Meflin-low ( }<20 \% \text { Meflin }+ \text { stromal cells) group }\end{array}$ \\
\hline Zhang et al., 2017 & Asporin & Breast cancer & $\begin{array}{l}\operatorname{HC}(n=60) \\
\operatorname{mRNA}(n=375)\end{array}$ & $\begin{array}{l}1 \text { Asporin is low expressed in TNBC and HER2 + tumors, compared with HR }+ \text { tumor } \\
2 \text { Low asporin express indicated to poor outcome, high asporin expression indicated a favorable } \\
\text { outcome }\end{array}$ \\
\hline Ono et al., 2013 & CD271 & $\begin{array}{l}\text { Pancreatic } \\
\text { cancer }\end{array}$ & $\mathrm{IHC}(n=105)$ & $\begin{array}{l}1 \text { CD271 + stromal expression was mostly detected on the tumor edge and was mainly in CAFs } \\
2 \text { CD271 + CAFs predominantly exist in the areas with strong a-SMA expression in the tumor } \\
3 \text { Stromal high CD271 expression represented a better prognosis. }\end{array}$ \\
\hline Wang et al., 2017 & Cav-1 & Breast cancer & $\Perp \mathrm{HC}(n=358)$ & $\begin{array}{l}1 \text { Cav-1 was predominantly expressed in CAFs in breast cancer } \\
2 \text { Cav-1-positive breast cancer patients showed increased cancer-specific survival compared } \\
\text { Cav-1-negative group } \\
3 \text { Cav-1-deficient CAFs enhanced the invasiveness of breast cancer cells. }\end{array}$ \\
\hline
\end{tabular}

Abbreviations: HR, hormone receptor; IHC, Immunohistochemistry; ISH, In situ hybridization; and TNBC, triple-negative breast cancer.

the expression of Cav-1 undergo a series of metabolic changes and autophagy. The process of autophagy in CAFs stimulates mitochondrial activity in adjacent tumor cells by providing a critical source of energy-rich glutamine (Nwosu et al., 2016). In a retrospective study regarding breast cancer patients, the Cav-1-positive group showed 72 months of cancer-specific survival, whereas the survival time of the Cav-1-negative group was 29.5 months (Simpkins et al., 2012). Cav-1 negative tumors show increased tumor progression, metastasis, and estrogen receptor-negative genotype compared to Cav-1 positive breast cancer (Sloan et al., 2009; Witkiewicz et al., 2009; Qian et al., 2011; Simpkins et al., 2012). Asporin is another tumor inhibitor protein expressed by CAFs in breast cancer. A histopathological study on human breast cancer $(n=180)$ indicated that asporin has low expression in TNBC and HER2 + tumors, which are both aggressive breast cancer types. Survival analysis suggested that low asporin status is an independent risk factor correlated to poor outcome, whereas high asporin expression indicated a favorable outcome (Maris et al., 2015). CD271, a neurotrophin receptor, is also named as the nerve growth factor receptor (NGFR; Liang et al., 2018; Nielsen et al., 2018). The prognostic value of stromal CD271 was assessed in 31 normal pancreases and 105 pancreatic cancer (PDACs) by an IHC assay. Stromal CD271 was express mainly in CAFs, and its high expression represented a better prognosis. Results suggested CD271 + CAFs acts to restrain cancer progression. Interestingly, CD271 + CAFs predominantly existed in the areas with strong a-SMA expression in the tumor, which suggested CD271 + CAFs was a subpopulation of SMA + CAFs. Yet, how SMA + CAFs differentiated into CD271 + CAFs and CD271CAFs is unknown, and in pancreatic cancer, the functional role of CD271 + CAFs remains to be explored (Fujiwara et al., 2012). In another PDACs related study, approximately $10 \%$ of a-SMA + CAFs showed positive Meflin mRNA expression in In situ hybridization (ISH) assay. The PDACs tissue samples were divided into Meflin-high ( $\geq 20 \%$ Meflin + stromal cells) and Meflinlow ( $<20 \%$ Meflin + stromal cells) groups. The Meflinhigh group exhibited better prognosis and more differentiated histology than the Meflin-low group, which indicated that Meflin expression in CAFs correlates with a favorable outcome of human patients with PDAC (Mizutani et al., 2019).

\section{SINGLE-CELL RNA-SEQUENCING: A NEW STRATEGY TO CLASSIFY CAFS SUBPOPULATION}

The coexistence of tumor restraining and tumor-promoting abilities within the CAFs population seems to be puzzling. These contradictory results can be explained by the existence of CAFs subpopulations with opposing functions. Therefore, it is essential to classify CAFs by combining a couple of different markers to identify their biological characteristics, thereby improving their therapeutic relevance. Using Single-cell RNA-sequencing, we can examine the transcriptome of single cells to distinguish cell subpopulations inferred by the same transcriptional programs. Single-cell RNA-sequencing allows us to recognize fibroblasts subsets within CAFs by restricting analytical cell numbers (Bartoschek et al., 2018). Li H et al analyzed CAFs from human CRC by single-cell RNA sequencing. Based on TGF $\beta$ signaling gene expression, CAFs in colorectal cancer were divided into two major subtypes: CAF-As and CAF-Bs. The CAF-As showed a high expression of matrix collagen type I $\alpha 2$ (COL1A2), decorin, metalloproteinase 2 (MMP2). At the same time, CAF-Bs exhibited high expression of myofibroblastic markers such as $\alpha \mathrm{SMA}$, transgelin, and PDGF$\alpha$ (Li H. et al., 2017). Another study applied single-cell RNA sequencing to explore CAFs from the pancreatic cancer sample. Results verified the existence of previously reported myCAF and iCAF subpopulations (Ohlund et al., 2017) and mapped the gene signatures of these CAFs subsets. Notably, in this study, a new subpopulation of CAFs named "antigen-presenting CAFs" (apCAF) was identified. This subtype CAFs expressed MHC class II (MHCII)-related genes and presented antigens to CD $4+$ T cells, which regulating immune response (Elyada et al., 2019). The above studies provide evidence that single-cell RNAsequencing helps to map the gene signature and function of CAFs subpopulations. However, we are still in the beginning to take 
advantage of this technique to explain the origin, maker, function, and intratumoral heterogeneity in CAFs.

\section{DISCUSSION}

This review summarizes the primary evidence of CAFs' protective role against cancer. Although the hypotheses of a guarding stroma against cancer are not novel, we now possess a more robust picture of CAFs possible tumor restraining reactions. The surprising results that CAFs' depletion in transgenic mice models accelerate tumor progression raise the cautionof nonspecific CAFs depletion target therapy. Theoretically, patients can benefit from a successful anti-CAFs therapy as: (1) targeting pCAFs or the pCAFs-releasing factors; (2) normalize pCAFs to NFs; and (3) reprogramming of pCAFs to rCAFs phenotype (Sahai et al., 2020). A good example of normalizing CAFs is targeting the vitamin $\mathrm{D}$ receptor in pancreatic cancer. Vitamin D receptor ligand calcipotriol treatment reverted CAFs activation state into a quiescent state. This lead to an increased intratumoral gemcitabine concentration and prolonged survival time (Sherman et al., 2014). Given the reversible CAFs functions and subtypes, targeting pCAFs or reprogramming of pCAFs to rCAFs remains a challenge for the field. It is critically important to identify the individual fibroblast's state in the CAFs population and pre-existing "lineage-restricted" effects that control CAFs phynotypes (Sahai et al., 2020).

Recently, the interaction between CAFs and immune cells in the TME has been increasingly recognized. Many studies in this field conclude that CAFs inhibit host anti-tumor immunity by shaping the immune cells, such as monocytes or neutrophils, into an immunosuppressive phenotype. By contrast, limited results are showing CAFs suppress tumor progression by enhancing host immunity (Ziani et al., 2018). Based on the above evidence of CAFs' tumor inhibition effects, we believe that a particular CAFs subtype, which can strengthen host anti-tumor immunity might exist in the early cancer stage. A more delicate co-culture system needs to established and explore the cross-talk between tumor cells, CAFs subtype, and immune cells.

Fundamentally, CAFs biology will be best understood through subtyping by biology and by function. Single-cell sequencing provides a new insight to map the functional role of distinct CAFs types. To examine the functional associations among the diverse CAFs subpopulation, we now urgently require generating

\section{REFERENCES}

Albrengues, J., Bertero, T., Grasset, E., Bonan, S., Maiel, M., Bourget, I., et al. (2015). Epigenetic switch drives the conversion of fibroblasts into proinvasive cancer-associated fibroblasts. Nat. Commun. 6:10204.

Arnold, J. N., Magiera, L., Kraman, M., and Fearon, D. T. (2014). Tumoral immune suppression by macrophages expressing fibroblast activation protein-alpha and heme oxygenase-1. Cancer Immunol. Res. 2, 121-126. doi: 10.1158/2326-6066. cir-13-0150

Bartoschek, M., Oskolkov, N., Bocci, M., Lovrot, J., Larsson, C., Sommarin, M., et al. (2018). Spatially and functionally distinct subclasses of breast cancerassociated fibroblasts revealed by single cell RNA sequencing. Nat. Commun. 9:5150. computational tools for cross-platform comparison. Notably, the CAFs' tumor-promoting or tumor-restraining status can convert to each other depending on different tumor stages. CAFs population lineage tracing provides a useful approach to demonstrate the evolution between different CAFs subset. Accordingly, we need to figure out which factors from cancer cells or other stromal cells determine the signaling pathways of pCAFs and rCAFs and maintain their phenotype. Despite the vital research value of CAFs, our development of CAFsoriented cancer management approaches is still in the elemental stage. Shortly, we will have a deeper understanding of the genetic events and signaling changes in the evolution of CAFs. The emergence of a broadly accepted CAF molecular subtyping will better recognize CAFs' biological behaviors in particular contexts. These novel insights will help translate CAFs biology from bench to bedside and develop new strategies to improve precision cancer therapy.

\section{AUTHOR CONTRIBUTIONS}

WY and BY made substantial contributions to conception and determined the final version. ZW drafted the manuscript or revised it critically for important intellectual content. YaT, YiT, and JY contributed to table editing, figure drawing, and manuscript drafting. QY contributed to revising the manuscript by literature reviewing and drafting new parts of the manuscript. All authors read and approved the final manuscript.

\section{FUNDING}

This work was supported by grants from Zhejiang Provincial Natural Science Foundation of China (Natural Science Foundation of Zhejiang Province LY20H180015 to ZW). The sponsors of the study had no role in study design, data collection, data analysis, results in interpretation, writing the manuscript, and the decision to submit the manuscript for publication.

\section{ACKNOWLEDGMENTS}

We thank Dr. Weidong Chai from the Geroge Washington University for his essential work in English language editing.

Becker, A., Thakur, B. K., Weiss, J. M., Kim, H. S., Peinado, H., and Lyden, D. (2016). Extracellular vesicles in cancer: cell-to-cell mediators of metastasis. Cancer Cell 30, 836-848. doi: 10.1016/j.ccell.2016.10.009

Biswas, S., Davis, H., Irshad, S., Sandberg, T., Worthley, D., and Leedham, S. (2015). Microenvironmental control of stem cell fate in intestinal homeostasis and disease. J. Pathol. 237, 135-145. doi: 10.1002/path.4563

Calon, A., Lonardo, E., Berenguer-Llergo, A., Espinet, E., Hernando-Momblona, X., Iglesias, M., et al. (2015). Stromal gene expression defines poor-prognosis subtypes in colorectal cancer. Nat. Genet. 47, 320-329. doi: 10.1038/ng.3225

Chang, P. H., Hwang-Verslues, W. W., Chang, Y. C., Chen, C. C., Hsiao, M., Jeng, Y. M., et al. (2012). Activation of robol signaling of breast cancer cells by Slit2 from stromal fibroblast restrains tumorigenesis via blocking PI3K/Akt/betacatenin pathway. Cancer Res. 72, 4652-4661. 
Costa, A., Kieffer, Y., Scholer-Dahirel, A., Pelon, F., Bourachot, B., Cardon, M., et al. (2018). Fibroblast heterogeneity and immunosuppressive environment in human breast Cancer. Cancer Cell 33, 463-479.e10.

Curry, J. M., Tuluc, M., Whitaker-Menezes, D., Ames, J. A., Anantharaman, A., Butera, A., et al. (2013). Cancer metabolism, stemness and tumor recurrence: MCT1 and MCT4 are functional biomarkers of metabolic symbiosis in head and neck cancer. Cell Cycle 12, 1371-1384. doi: 10.4161/cc.24092

Dai, K., Tanaka, M., Kamiyoshi, A., Sakurai, T., Ichikawa-Shindo, Y., Kawate, H., et al. (2019). Deficiency of the adrenomedullin-RAMP3 system suppresses metastasis through the modification of cancer-associated fibroblasts. Oncogene 39, 1914-1930. doi: 10.1038/s41388-019-1112-z

Djurec, M., Grana, O., Lee, A., Troule, K., Espinet, E., Cabras, L., et al. (2018). $\mathrm{Saa} 3$ is a key mediator of the protumorigenic properties of cancer-associated fibroblasts in pancreatic tumors. Proc. Natl. Acad. Sci. U.S.A. 115, E1147E1156.

Elyada, E., Bolisetty, M., Laise, P., Flynn, W. F., Courtois, E. T., Burkhart, R. A., et al. (2019). Cross-species single-cell analysis of pancreatic ductal adenocarcinoma reveals antigen-presenting cancer-associated fibroblasts. Cancer Discov. 9, 1102-1123. doi: 10.1158/2159-8290.cd-19-0094

Erez, N., Truitt, M., Olson, P., Arron, S. T., and Hanahan, D. (2010). Cancerassociated fibroblasts are activated in incipient neoplasia to orchestrate tumorpromoting inflammation in an NF-kappaB-dependent manner. Cancer Cell 17, 135-147. doi: 10.1016/j.ccr.2009.12.041

Fang, A., Zhou, S., Su, X., Liu, C., Chen, X., Wan, Y., et al. (2018). RAMP3 is a prognostic indicator of liver cancer and might reduce the adverse effect of TP53 mutation on survival. Future Oncol. 14, 2615-2625. doi: 10.2217/fon-20180296

Feig, C., Jones, J. O., Kraman, M., Wells, R. J., Deonarine, A., Chan, D. S., et al. (2013). Targeting CXCL12 from FAP-expressing carcinoma-associated fibroblasts synergizes with anti-PD-L1 immunotherapy in pancreatic cancer. Proc. Natl. Acad. Sci. U.S.A. 110, 20212-20217. doi: 10.1073/pnas.1320318110

Fischer, K. R., Durrans, A., Lee, S., Sheng, J., Li, F., Wong, S. T., et al. (2015). Epithelial-to-mesenchymal transition is not required for lung metastasis but contributes to chemoresistance. Nature 527, 472-476. doi: 10.1038/ nature 15748

Fujiwara, K., Ohuchida, K., Mizumoto, K., Shindo, K., Eguchi, D., Kozono, S., et al. (2012). CD271(+) subpopulation of pancreatic stellate cells correlates with prognosis of pancreatic cancer and is regulated by interaction with cancer cells. PLoS One 7:e52682. doi: 10.1371/journal.pone.0052682

Gerling, M., Buller, N. V., Kirn, L. M., Joost, S., Frings, O., Englert, B., et al. (2016). Stromal hedgehog signalling is downregulated in colon cancer and its restoration restrains tumour growth. Nat. Commun. 7:12321.

Giatromanolaki, A., Koukourakis, M. I., Koutsopoulos, A., Mendrinos, S., and Sivridis, E. (2012). The metabolic interactions between tumor cells and tumorassociated stroma (TAS) in prostatic cancer. Cancer Biol. Ther. 13, 1284-1289. doi: $10.4161 /$ cbt. 21785

Giusti, I., Di Francesco, M., D’ascenzo, S., Palmerini, M. G., Macchiarelli, G., Carta, G., et al. (2018). Ovarian cancer-derived extracellular vesicles affect normal human fibroblast behavior. Cancer Biol. Ther. 19, 722-734.

Greten, F. R., Eckmann, L., Greten, T. F., Park, J. M., Li, Z. W., Egan, L. J., et al. (2004). IKKbeta links inflammation and tumorigenesis in a mouse model of colitis-associated cancer. Cell 118, 285-296. doi: 10.1016/j.cell.2004.07.013

Hanahan, D., and Weinberg, R. A. (2011). Hallmarks of cancer: the next generation. Cell 144, 646-674. doi: 10.1016/j.cell.2011.02.013

Ishii, G., Ochiai, A., and Neri, S. (2015). Phenotypic and functional heterogeneity of cancer-associated fibroblast within the tumor microenvironment. Adv. Drug Deliv. Rev. 99, 186-196. doi: 10.1016/j.addr.2015.07.007

Ito, M., Ishii, G., Nagai, K., Maeda, R., Nakano, Y., and Ochiai, A. (2012). Prognostic impact of cancer-associated stromal cells in patients with stage I lung adenocarcinoma. Chest 142, 151-158. doi: 10.1378/chest.11-2458

Iwano, M., Fischer, A., Okada, H., Plieth, D., Xue, C., Danoff, T. M., et al. (2001). Conditional abatement of tissue fibrosis using nucleoside analogs to selectively corrupt DNA replication in transgenic fibroblasts. Mol. Ther. 3, 149-159. doi: $10.1006 / \mathrm{mthe} .2000 .0251$

Kalluri, R. (2016). The biology and function of fibroblasts in cancer. Nat. Rev. Cancer 16, 582-598. doi: 10.1038/nrc.2016.73

Kizawa, H., Kou, I., Iida, A., Sudo, A., Miyamoto, Y., Fukuda, A., et al. (2005). An aspartic acid repeat polymorphism in asporin inhibits chondrogenesis and increases susceptibility to osteoarthritis. Nat. Genet. 37, 138-144. doi: 10.1038/ ng1496

Kobayashi, H., Enomoto, A., Woods, S. L., Burt, A. D., Takahashi, M., and Worthley, D. L. (2019). Cancer-associated fibroblasts in gastrointestinal cancer. Nat. Rev. Gastroenterol. Hepatol. 16, 282-295. doi: 10.1038/s41575-019-0115-0

Koliaraki, V., Pallangyo, C. K., Greten, F. R., and Kollias, G. (2017). Mesenchymal cells in colon cancer. Gastroenterology 152, 964-979.

Koliaraki, V., Pasparakis, M., and Kollias, G. (2015). IKKbeta in intestinal mesenchymal cells promotes initiation of colitis-associated cancer. J. Exp. Med. 212, 2235-2251. doi: 10.1084/jem.20150542

Li, B. L., Lu, W., Qu, J. J., Ye, L., Du, G. Q., and Wan, X. P. (2019). Loss of exosomal miR-148b from cancer-associated fibroblasts promotes endometrial cancer cell invasion and cancer metastasis. J. Cell Physiol. 234, 2943-2953. doi: $10.1002 /$ jcp. 27111

Li, H., Courtois, E. T., Sengupta, D., Tan, Y., Chen, K. H., Goh, J. J. L., et al. (2017). Reference component analysis of single-cell transcriptomes elucidates cellular heterogeneity in human colorectal tumors. Nat. Genet. 49, 708-718. doi: 10.1038/ng.3818

Li, L., Piontek, K., Ishida, M., Fausther, M., Dranoff, J. A., Fu, R., et al. (2017). Extracellular vesicles carry microRNA-195 to intrahepatic cholangiocarcinoma and improve survival in a rat model. Hepatology 65, 501-514. doi: 10.1002/hep. 28735

Li, Y. Y., Tao, Y. W., Gao, S., Li, P., Zheng, J. M., Zhang, S. E., et al. (2018). Cancerassociated fibroblasts contribute to oral cancer cells proliferation and metastasis via exosome-mediated paracrine miR-34a-5p. EBioMedicine 36, 209-220. doi: 10.1016/j.ebiom.2018.09.006

Liang, L., Coudiere-Morrison, L., Tatari, N., Stromecki, M., Fresnoza, A., Porter, C. J., et al. (2018). CD271(+) cells are diagnostic and prognostic and exhibit elevated MAPK activity in SHH medulloblastoma. Cancer Res. 78, 4745-4759. doi: 10.1158/0008-5472.can-18-0027

Liao, Y., Ni, Y., He, R., Liu, W., and Du, J. (2013). Clinical implications of fibroblast activation protein-alpha in non-small cell lung cancer after curative resection: a new predictor for prognosis. J. Cancer Res. Clin. Oncol. 139, 1523-1528. doi: 10.1007/s00432-013-1471-8

Luga, V., Zhang, L., Viloria-Petit, A. M., Ogunjimi, A. A., Inanlou, M. R., Chiu, E., et al. (2012). Exosomes mediate stromal mobilization of autocrine Wnt-PCP signaling in breast cancer cell migration. Cell 151, 1542-1556. doi: 10.1016/j. cell.2012.11.024

Maacha, S., Bhat, A. A., Jimenez, L., Raza, A., Haris, M., Uddin, S., et al. (2019). Extracellular vesicles-mediated intercellular communication: roles in the tumor microenvironment and anti-cancer drug resistance. Mol. Cancer 18:55.

Mackie, D. I., Nielsen, N. R., Harris, M., Singh, S., Davis, R. B., Dy, D., et al. (2019). RAMP3 determines rapid recycling of atypical chemokine receptor-3 for guided angiogenesis. Proc. Natl. Acad. Sci. U.S.A. 116, 24093-24099. doi: 10.1073/pnas.1905561116

Mao, J., Ligon, K. L., Rakhlin, E. Y., Thayer, S. P., Bronson, R. T., Rowitch, D., et al. (2006). A novel somatic mouse model to survey tumorigenic potential applied to the hedgehog pathway. Cancer Res. 66, 10171-10178. doi: 10.1158/00085472.can-06-0657

Maris, P., Blomme, A., Palacios, A. P., Costanza, B., Bellahcene, A., Bianchi, E., et al. (2015). Asporin is a fibroblast-derived TGF-betal inhibitor and a tumor suppressor associated with good prognosis in breast cancer. PLoS Med. 12:e1001871. doi: 10.1371/journal.pmed.1001871

Martinez-Outschoorn, U. E., Lisanti, M. P., and Sotgia, F. (2014). Catabolic cancerassociated fibroblasts transfer energy and biomass to anabolic cancer cells, fueling tumor growth. Semin. Cancer Biol. 25, 47-60. doi: 10.1016/j.semcancer. 2014.01.005

Martinez-Outschoorn, U. E., Whitaker-Menezes, D., Lin, Z., Flomenberg, N., Howell, A., Pestell, R. G., et al. (2011). Cytokine production and inflammation drive autophagy in the tumor microenvironment: role of stromal caveolin1 as a key regulator. Cell Cycle 10, 1784-1793. doi: 10.4161/cc.10.11. 15674

Mclatchie, L. M., Fraser, N. J., Main, M. J., Wise, A., Brown, J., Thompson, N., et al. (1998). RAMPs regulate the transport and ligand specificity of the calcitonin-receptor-like receptor. Nature 393, 333-339. doi: 10.1038/30666

Mizutani, Y., Kobayashi, H., Iida, T., Asai, N., Masamune, A., Hara, A., et al. (2019). Meflin-positive cancer-associated fibroblasts inhibit pancreatic carcinogenesis. Cancer Res. 79, 5367-5381. doi: 10.1158/0008-5472.can-19-0454 
Monti, D., Sotgia, F., Whitaker-Menezes, D., Tuluc, M., Birbe, R., Berger, A., et al. (2017). Pilot study demonstrating metabolic and anti-proliferative effects of in vivo anti-oxidant supplementation with $\mathrm{N}$-acetylcysteine in breast cancer. Semin. Oncol. 44, 226-232. doi: 10.1053/j.seminoncol.2017.10.001

Morton, J. P., Mongeau, M. E., Klimstra, D. S., Morris, J. P., Lee, Y. C., Kawaguchi, Y., et al. (2007). Sonic hedgehog acts at multiple stages during pancreatic tumorigenesis. Proc. Natl. Acad. Sci. U.S.A. 104, 5103-5108. doi: 10.1073/pnas. 0701158104

Mueller, M. M., and Fusenig, N. E. (2004). Friends or foes - bipolar effects of the tumour stroma in cancer. Nat. Rev. Cancer 4, 839-849. doi: 10.1038/nrc1477

Nielsen, P. S., Riber-Hansen, R., and Steiniche, T. (2018). Immunohistochemical CD271 expression correlates with melanoma progress in a case-control study. Pathology 50, 402-410. doi: 10.1016/j.pathol.2017.12.340

Nwosu, Z. C., Ebert, M. P., Dooley, S., and Meyer, C. (2016). Caveolin-1 in the regulation of cell metabolism: a cancer perspective. Mol. Cancer 15:71.

Ohlund, D., Elyada, E., and Tuveson, D. (2014). Fibroblast heterogeneity in the cancer wound. J. Exp. Med. 211, 1503-1523. doi: 10.1084/jem.20140692

Ohlund, D., Handly-Santana, A., Biffi, G., Elyada, E., Almeida, A. S., PonzSarvise, M., et al. (2017). Distinct populations of inflammatory fibroblasts and myofibroblasts in pancreatic cancer. J. Exp. Med. 214, 579-596. doi: 10.1084/ jem.20162024

Ono, S., Ishii, G., Nagai, K., Takuwa, T., Yoshida, J., Nishimura, M., et al. (2013). Podoplanin-positive cancer-associated fibroblasts could have prognostic value independent of cancer cell phenotype in stage I lung squamous cell carcinoma: usefulness of combining analysis of both cancer cell phenotype and cancerassociated fibroblast phenotype. Chest 143, 963-970. doi: 10.1378/chest.120913

Osterreicher, C. H., Penz-Osterreicher, M., Grivennikov, S. I., Guma, M., Koltsova, E. K., Datz, C., et al. (2011). Fibroblast-specific protein 1 identifies an inflammatory subpopulation of macrophages in the liver. Proc. Natl. Acad. Sci. U.S.A. 108, 308-313. doi: 10.1073/pnas.1017547108

Ozdemir, B. C., Pentcheva-Hoang, T., Carstens, J. L., Zheng, X., Wu, C. C., Simpson, T. R., et al. (2014). Depletion of carcinoma-associated fibroblasts and fibrosis induces immunosuppression and accelerates pancreas cancer with reduced survival. Cancer Cell 25, 719-734. doi: 10.1016/j.ccr.2014.04.005

Pallangyo, C. K., Ziegler, P. K., and Greten, F. R. (2015). IKKbeta acts as a tumor suppressor in cancer-associated fibroblasts during intestinal tumorigenesis. J. Exp. Med. 212, 2253-2266. doi: 10.1084/jem.20150576

Pasca Di Magliano, M., Sekine, S., Ermilov, A., Ferris, J., Dlugosz, A. A., and Hebrok, M. (2006). Hedgehog/Ras interactions regulate early stages of pancreatic cancer. Genes Dev. 20, 3161-3173. doi: 10.1101/gad.1470806

Paulsson, J., Sjoblom, T., Micke, P., Ponten, F., Landberg, G., Heldin, C. H., et al. (2009). Prognostic significance of stromal platelet-derived growth factor betareceptor expression in human breast cancer. Am. J. Pathol. 175, 334-341. doi: 10.2353/ajpath.2009.081030

Proia, D. A., and Kuperwasser, C. (2005). Stroma: tumor agonist or antagonist. Cell Cycle 4, 1022-1025. doi: 10.4161/cc.4.8.1903

Qian, N., Ueno, T., Kawaguchi-Sakita, N., Kawashima, M., Yoshida, N., Mikami, Y., et al. (2011). Prognostic significance of tumor/stromal caveolin-1 expression in breast cancer patients. Cancer Sci. 102, 1590-1596. doi: 10.1111/j.1349-7006. 2011.01985.x

Rhim, A. D., Mirek, E. T., Aiello, N. M., Maitra, A., Bailey, J. M., Mcallister, F., et al. (2012). EMT and dissemination precede pancreatic tumor formation. Cell 148, 349-361. doi: 10.1016/j.cell.2011.11.025

Rhim, A. D., Oberstein, P. E., Thomas, D. H., Mirek, E. T., Palermo, C. F., Sastra, S. A., et al. (2014). Stromal elements act to restrain, rather than support, pancreatic ductal adenocarcinoma. Cancer Cell 25, 735-747. doi: 10.1016/j.ccr. 2014.04.021

Richards, K. E., Zeleniak, A. E., Fishel, M. L., Wu, J., Littlepage, L. E., and Hill, R. (2017). Cancer-associated fibroblast exosomes regulate survival and proliferation of pancreatic cancer cells. Oncogene 36, 1770-1778. doi: 10.1038/ onc. 2016.353

Roberts, K. J., Kershner, A. M., and Beachy, P. A. (2017). The stromal niche for epithelial stem cells: a template for regeneration and a brake on malignancy. Cancer Cell 32, 404-410. doi: 10.1016/j.ccell.2017.08.007

Sahai, E., Astsaturov, I., Cukierman, E., Denardo, D. G., Egeblad, M., Evans, R. M., et al. (2020). A framework for advancing our understanding of cancerassociated fibroblasts. Nat. Rev. Cancer 20, 174-186. doi: 10.1038/s41568-0190238-1
Salomon, B., Maury, S., Loubiere, L., Caruso, M., Onclercq, R., and Klatzmann, D. (1995). A truncated herpes simplex virus thymidine kinase phosphorylates thymidine and nucleoside analogs and does not cause sterility in transgenic mice. Mol. Cell Biol. 15, 5322-5328. doi: 10.1128/mcb.15.10. 5322

Satoyoshi, R., Kuriyama, S., Aiba, N., Yashiro, M., and Tanaka, M. (2015). Asporin activates coordinated invasion of scirrhous gastric cancer and cancer-associated fibroblasts. Oncogene 34, 650-660. doi: 10.1038/onc.2013.584

Seoane, J., and Gomis, R. R. (2017). TGF-beta family signaling in tumor suppression and cancer progression. Cold Spring Harb. Perspect. Biol. 9:a022277. doi: 10.1101/cshperspect.a022277

Sherman, M. H., Yu, R. T., Engle, D. D., Ding, N., Atkins, A. R., Tiriac, H., et al. (2014). Vitamin D receptor-mediated stromal reprogramming suppresses pancreatitis and enhances pancreatic cancer therapy. Cell 159, 80-93. doi: 10.1016/j.cell.2014.08.007

Simpkins, S. A., Hanby, A. M., Holliday, D. L., and Speirs, V. (2012). Clinical and functional significance of loss of caveolin-1 expression in breast cancer-associated fibroblasts. J. Pathol. 227, 490-498. doi: 10.1002/path. 4034

Sloan, E. K., Ciocca, D. R., Pouliot, N., Natoli, A., Restall, C., Henderson, M. A., et al. (2009). Stromal cell expression of caveolin-1 predicts outcome in breast cancer. Am. J. Pathol. 174, 2035-2043. doi: 10.2353/ajpath.2009. 080924

Sotgia, F., Martinez-Outschoorn, U. E., Pavlides, S., Howell, A., Pestell, R. G., and Lisanti, M. P. (2011). Understanding the Warburg effect and the prognostic value of stromal caveolin-1 as a marker of a lethal tumor microenvironment. Breast Cancer Res. 13:213.

Sugimoto, H., Mundel, T. M., Kieran, M. W., and Kalluri, R. (2006). Identification of fibroblast heterogeneity in the tumor microenvironment. Cancer Biol. Ther. 5, 1640-1646. doi: 10.4161/cbt.5.12.3354

Theiss, A. L., Simmons, J. G., Jobin, C., and Lund, P. K. (2005). Tumor necrosis factor (TNF) alpha increases collagen accumulation and proliferation in intestinal myofibroblasts via TNF receptor 2. J. Biol. Chem. 280, 36099-36109. doi: 10.1074/jbc.m505291200

Ullah, M. S., Davies, A. J., and Halestrap, A. P. (2006). The plasma membrane lactate transporter MCT4, but not MCT1, is up-regulated by hypoxia through a HIF-1alpha-dependent mechanism. J. Biol. Chem. 281, 9030-9037. doi: 10. 1074/jbc.m511397200

Valenti, G., Quinn, H. M., Heynen, G., Lan, L., Holland, J. D., Vogel, R., et al. (2017). Cancer stem cells regulate cancer-associated fibroblasts via activation of hedgehog signaling in mammary gland tumors. Cancer Res. 77, 2134-2147. doi: 10.1158/0008-5472.can-15-3490

Wallace, D. C. (2012). Mitochondria and cancer. Nat. Rev. Cancer 12, 685-698. doi: $10.1038 / \operatorname{nrc} 3365$

Wang, Z., Tan, Y., Yu, W., Zheng, S., Zhang, S., Sun, L., et al. (2017). Small role with big impact: miRNAs as communicators in the cross-talk between cancer-associated fibroblasts and cancer cells. Int. J. Biol. Sci. 13, 339-348. doi: 10.7150/ijbs. 17680

Webber, J. P., Spary, L. K., Sanders, A. J., Chowdhury, R., Jiang, W. G., Steadman, R., et al. (2015). Differentiation of tumour-promoting stromal myofibroblasts by cancer exosomes. Oncogene 34, 290-302. doi: 10.1038/onc.2013.560

Whitaker-Menezes, D., Martinez-Outschoorn, U. E., Lin, Z., Ertel, A., Flomenberg, N., Witkiewicz, A. K., et al. (2011). Evidence for a stromal-epithelial "lactate shuttle" in human tumors: MCT4 is a marker of oxidative stress in cancerassociated fibroblasts. Cell Cycle 10, 1772-1783. doi: 10.4161/cc.10.11.15659

Witkiewicz, A. K., Dasgupta, A., Sotgia, F., Mercier, I., Pestell, R. G., Sabel, M., et al. (2009). An absence of stromal caveolin-1 expression predicts early tumor recurrence and poor clinical outcome in human breast cancers. Am. J. Pathol. 174, 2023-2034.

Witkiewicz, A. K., Whitaker-Menezes, D., Dasgupta, A., Philp, N. J., Lin, Z., Gandara, R., et al. (2012). Using the "reverse Warburg effect" to identify highrisk breast cancer patients: stromal MCT4 predicts poor clinical outcome in triple-negative breast cancers. Cell Cycle 11, 1108-1117. doi: 10.4161/cc.11.6. 19530

Worthley, D. L., Churchill, M., Compton, J. T., Tailor, Y., Rao, M., Si, Y., et al. (2015). Gremlin 1 identifies a skeletal stem cell with bone, cartilage, and reticular stromal potential. Cell 160, 269-284. doi: 10.1016/j.cell.2014.11.042

Xu, G., Zhang, B., Ye, J., Cao, S., Shi, J., Zhao, Y., et al. (2019). Exosomal miRNA-139 in cancer-associated fibroblasts inhibits gastric cancer progression 
by repressing MMP11 expression. Int. J. Biol. Sci. 15, 2320-2329. doi: 10.7150/ ijbs. 33750

Yang, L., Achreja, A., Yeung, T. L., Mangala, L. S., Jiang, D., Han, C., et al. (2016). Targeting stromal glutamine synthetase in tumors disrupts tumor microenvironment-regulated cancer cell growth. Cell Metab. 24, 685-700. doi: 10.1016/j.cmet.2016.10.011

Zhang, D., Wang, Y., Shi, Z., Liu, J., Sun, P., Hou, X., et al. (2015). Metabolic reprogramming of cancer-associated fibroblasts by IDH3alpha downregulation. Cell Rep. 10, 1335-1348. doi: 10.1016/j.celrep.2015.02.006

Zhang, J., Chen, L., Liu, X., Kammertoens, T., Blankenstein, T., and Qin, Z. (2013). Fibroblast-specific protein 1/S100A4-positive cells prevent carcinoma through collagen production and encapsulation of carcinogens. Cancer Res. 73, 2770-2781. doi: 10.1158/0008-5472.CAN-12-3022

Zhang, Y., Wei, J., Xu, J., Leong, W. S., Liu, G., Ji, T., et al. (2018). Suppression of tumor energy supply by liposomal nanoparticle-mediated inhibition of aerobic glycolysis. ACS Appl. Mater. Interfaces 10, 2347-2353. doi: 10.1021/acsami. $7 \mathrm{~b} 16685$
Zhang, Z., Li, X., Sun, W., Yue, S., Yang, J., Li, J., et al. (2017). Loss of exosomal miR-320a from cancer-associated fibroblasts contributes to HCC proliferation and metastasis. Cancer Lett. 397, 33-42. doi: 10.1016/j.canlet.2017.03.004

Ziani, L., Chouaib, S., and Thiery, J. (2018). Alteration of the antitumor immune response by cancer-associated fibroblasts. Front. Immunol. 9:414. doi: 10.3389/ fimmu.2018.00414

Conflict of Interest: The authors declare that the research was conducted in the absence of any commercial or financial relationships that could be construed as a potential conflict of interest.

Copyright $\odot 2021$ Wang, Yang, Tan, Tang, Ye, Yuan and Yu. This is an open-access article distributed under the terms of the Creative Commons Attribution License (CC BY). The use, distribution or reproduction in other forums is permitted, provided the original author(s) and the copyright owner(s) are credited and that the original publication in this journal is cited, in accordance with accepted academic practice. No use, distribution or reproduction is permitted which does not comply with these terms. 